\title{
Stream Cipher by Reed-Solomon Codes
}

\author{
Tao $\mathrm{Wu}^{\Uparrow}{ }^{1,2}$, Ruomei Wang ${ }^{1}$ \\ 1 School of Data and Computer Science, Sun Yat-Sen University, Guangzhou 510006, P.R. China \\ 2 Shenzhen Research Institute of Sun Yat-Sen University, Shenzhen 518057, P.R. China
}

A R T I C L E I N F O

Article history:

Received: 04 May, 2018

Accepted: 07 July, 2018

Online: 14 July, 2018

Keywords:

Stream cipher

Reed-Solomon code

Public-key cryptography

\begin{abstract}
A B S T R A C T
Stream cipher can be used in constrained environments to provide information security and reduce energy expense at data transmission. In this paper, it is shown that Reed-Solomon (RS) code can be used to implement stream cipher, which is widely used for error corrections of data in transmission and storages. The proposed stream cipher combine the keys with the messages, and conceals the cipher within the erroneous RS code. Then at the receiving part the cipher can be checked out by $R S$ decoding, leaving the information unchanged. There are several advantages with this scheme: First, compared with the usual stream cipher including two engines, only one engine is required, making the synchronization of the stream cipher and messages easily. In the situation, the stream cipher and the messages are held as a whole while the alignment of stream cipher is not needed. Thus the usual power consumptions for synchronization and key generation at the receiving part can be saved. Second, the stream cipher increases the security level by adding the difficulty of decoding a random linear $R S$ code, which stores the key streams like public key cryptography. Since the RS encoding and decoding modules are already included in many computer systems or devices, the new scheme may be implemented by reconfiguration rather than extra hardware units. Compared with generalized RS code for code-based public-key cryptography like McEliece system, it uses systematic encoding instead of nonsystematic encoding, which decreases the power overhead. Unlike the encryption by public keys and decryption by private keys of McEliece system, it encrypts messages by private keys from stream cipher and decrypts texts by public keys implemented in the RS decoder.
\end{abstract}

\section{Introduction}

Stream cipher by Reed-Solomon (RS) code this work intends to implement encryption by encoding if necessary [1]. As the encoding-encryption paradigm has been used in the standard for mobile telephony GSM [2, 3], it has received much attention so far. RS code has been widely used in communications and storage systems to correct errors, which can also be used together with $\mathrm{BCH}$ code to ensure data integrity [4]. The idea of using RS code as a carriage for stream cipher comes from the observation of RS code for code-based cryptography, where it is found that the errors are intentionally inserted and recovered with much computation and energy [5, 6]. In fact, the code-based peudo random generator and its security can be considered to achieve stream cipher [7]. In [8], the generalized RS code is used to reduces the density of a transform matrix and improves weakly secure data exchange.

Stream cipher belongs to symmetric cryptography and can be built by many devices, such as linear and nonlinear feedback shift registers. For example, the Rakaposhi stream cipher consists of a 128-bit nonlinear feedback shift register, a 192-bit dynamic linear feedback shift register, and a nonlinear filter function [9]. Ubiquitous environments demands security, speed and power consciousness in processing huge amount of multimedia data [10], in which stream cipher can be used. Stream cipher is an engine that generates bit streams to mix the message. At the sending part, the

${ }^{*}$ Tao Wu, Shenzhen Institute of Industry-University-Research, Sun Yat-Sen University, email: nstrch@outlook.com 
plain text is confused with one bit stream, while at the receiving part, the cipher text is processed with the same bit stream again to recover the message. The stream cipher is like a determined peudo-random discrete function, of which the security can be analyzed by the generating mechanism of the cipher. A stream cipher can be marked by a key stream $(K)$ and the initialization vector $(I V)$, while the adversary may recover all the secret bits of $K$ after observing many related $(K, I V)$ pairs [9].

Usually, an one-way function and multiple steps are devised to safeguard the key generations or initialization processes, let bit XOR as the only operation to encrypt the message. By contrast, people also seek approaches to implement more secure code by public key cryptography. Code based cryptography [11] with Goppa codes [12] or MDPC codes [13], for example, can be applied to realize McEliece system . Meanwhile, generalized RS code can also be used to implement asymmetric cryptography [6], which brings out short keys compared with that obtained by Goppa code.

In this work, a stream cipher with RS code is proposed, in which the messages are encoded as RS codewords. This idea comes from its similarity to the configured logic with a DSP unit in FPGA. At the receiving part, the plaintexts and cipher are recovered from RS codes. The contributions of this paper include:

- a mechanism to include stream cipher in RS encoding and decoding, which is able to cover the message in a simple way.

- a review of related work about stream cipher of different schemes.

- a hardware implementation of stream cipher along by RS code.

The remaining parts of this paper is organized as follows: Sect.2 is a review of RS encoding and decoding; Sect. 3 briefly introduces the related work with stream cipher and code-based cryptography; Sect. 4 discusses the stream cipher along with RS code; then the hardware implementation and comparison of the stream cipher are given in Sect. 5, the last section concludes this paper.

\section{RS Code}

RS code is widely used for error corrections. The encoding can be either implemented by polynomial division with shift registers, or it can be performed by multiplying the generating matrix [14]. Suppose the RS code is defined over $G F\left(2^{m}\right)$ with $n$ code bits and $k$ information bits. Message sequence $\left(u_{k-1}, \ldots, u_{1}, u_{0}\right)$ is equivalent to

$$
u(X)=u_{0} \cdot X^{k-1}+u_{1} \cdot X^{k-2}+\cdots+u_{k-1} .
$$

Let the parity check code be $\left(v_{0}, v_{1}, \ldots, v_{n-k-1}\right)$, with $v(X)=X^{n-k} u(X) \bmod g(X)$, then the code polynomial yields

$c(X)=X^{n-k} u(X)+v_{0} \cdot X^{n-k-1}+v_{1} \cdot X^{n-k-2}+\cdots+v_{n-k-1}$,

where $u(X)=\sum_{i=0}^{k-1} u_{i} \cdot X^{k-1-i}, n-k=2 t$, and $t$ is the maximum number of error corrections. Then the encoded symbols will read $\left(v_{n-k-1}, \ldots, v_{0}, u_{k-1}, \ldots, u_{0}\right)$, with $v_{i}$ and $u_{i}$ being symbols in $G F\left(2^{m}\right)$.

Suppose the code generator is expressed as $g(X)=$ $\prod_{i=1}^{2 t}\left(X-\alpha^{i}\right)=g_{0}+g_{1} X+\cdots+g_{2 t-1} X^{2 t-1}+X^{2 t}$. Then $g_{0}=\prod_{i=1}^{2 t} \alpha^{i}, g_{1}=-\sum_{i=1}^{2 t} \prod_{j \neq i}^{1 \leqslant j \leqslant 2 t} \alpha^{j}$. For $2 \leqslant i \leqslant 2 t-1$, the generator polynomial can be computed in Galois field recursively. In Matlab, the generator polynomial can be computed directly by function rsgenpoly.

Once the generator is calculated, the generator matrix can be obtained. Take $n=255, k=223, t=16$, and $m=8$, then the generating matrix can be computed in Matlab as follows:

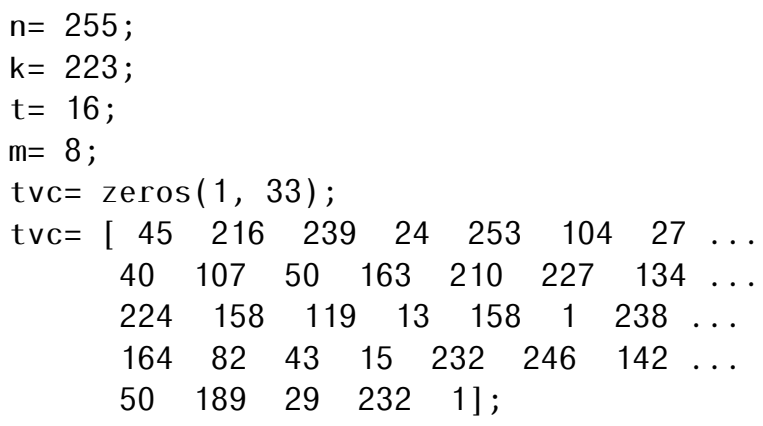

The encoding of RS code can be computed as modular division in the polynomial field [14]. Let $b(X)=X^{n-k} u(X) \bmod g(X)$, then $b(X)+X^{n-k} u(X)$ can be divided by $g(X)$ and become a RS code polynomial. The symbol $u_{0}$ with the lowest index is usually processed as the symbol with the highest weight for convenience.

The RS decoding is divided into four steps, i.e., syndrome computation, solution of error location polynomial, Chien search, and determination of the error values by Forney's algorithm. RS code is defined in the extension field $G F\left(2^{m \cdot s}\right)$, and syndromes can be 


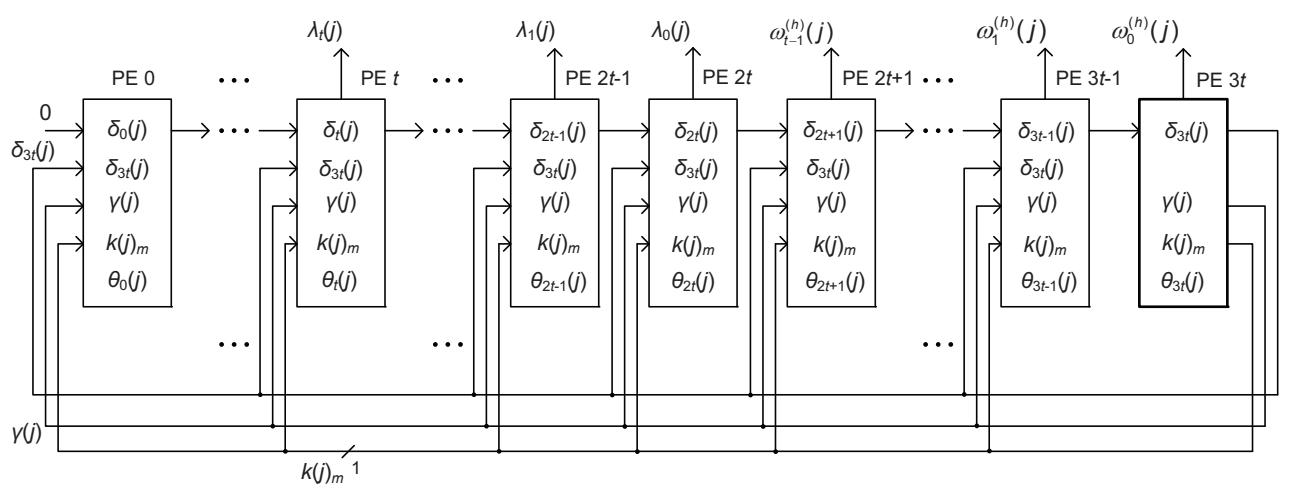

Figure 1: System architecture to solve error location polynomial [16, 1].

computed according to [15]:

$$
S_{i}=r\left(\alpha^{i}\right)=\sum_{j=0}^{n-1} r_{j} \cdot \alpha^{i \cdot j},
$$

where $r_{j} \in G F\left(2^{m}\right), 1 \leqslant i \leqslant 2 t$.

The error polynomial can be obtained by the improved inversion-less Berlekamp-Massey algorithm [16]. The resulting error polynomial reads

$$
\sigma(X)=\sum_{i=0}^{e} \lambda_{i} X^{i}
$$

where $v \leqslant t . \lambda_{i}$ is used to calculate the error locations. The error values are

$$
\begin{aligned}
Y_{i} & =\left.\frac{z^{2 t} \Omega^{(h)}(z)}{\sigma^{\prime}(z)}\right|_{z=X_{i}^{-1},} \\
& =\left.\frac{z^{2 t} \cdot \sum_{i=0}^{e-1} \omega_{i}^{(h)} \cdot z^{i}}{\sum_{i=2 k-1}^{k=1, \ldots,\left[\frac{e}{2}\right\rceil} \lambda_{i} \cdot z^{i-1}}\right|_{z=X_{i}^{-1},}
\end{aligned}
$$

where $\Omega^{(h)}(z)=\sum_{i=0}^{e-1} \omega_{i}^{(h)}(z) \cdot z^{i}$, the symbol $h$ denotes the higher partial products from $z^{2 t}$ to $z^{2 t+e-1}$, and $e$ is the number of error locations.

The system architecture of RS decoding [16, 1] is shown in Fig. 2. First, the original passages through processing elements are replaced by direct connections of signals. Second, the control logics are totaly integrated into the last processing element for simplicity. The signal $k(j)_{m}$ is the sign bit of the integer $k(j)$, where $m$ denotes the $m$-th bit. The coefficients from $\lambda_{t}$ to $\lambda_{0}$ come out of from PE $t$ to PE $2 t$, while the coefficients from $\omega_{t-1}^{(h)}$ to $\omega_{0}^{(h)}$ are produced from PE $2 t+1$ to PE $3 t$.

Among the processing elements, there exists matrix products. Typically, the matrix multiplication $\left[\alpha^{m}, \alpha^{m+1}, \ldots, \alpha^{2 m-2}\right]^{T}=Q \cdot\left[1, \alpha, \ldots, \alpha^{m-1}\right]^{T}$, the $G F\left(2^{m}\right)$ can be computed according to $C=A \cdot B=$ $\left(L+Q^{T} \cdot U\right) \cdot B$ proposed in [17]. In Matlab, the matrix $Q$ in $G F\left(2^{8}\right)$ can be obtained in the following:

$T x=\operatorname{zeros}(m-1, m)$;
$\operatorname{Ty} 1=\operatorname{zeros}(\mathrm{m}, \mathrm{m})$

$\operatorname{vf} 0=[1,0,1,1,1,0,0,0]$;

$\operatorname{Ty}(:, 1: \mathrm{m}-1)=[\operatorname{zeros}(1, \mathrm{~m}-1) ; \operatorname{eye}(\mathrm{m}-1)]$;

$\operatorname{Ty}(:, m)=\operatorname{vfO} .^{\prime}$;

$\mathrm{Ty} 1=\mathrm{Ty}^{\wedge} \mathrm{m}$;

$\operatorname{Ty} 1=\bmod (\operatorname{Ty} 1,2)$;

$\operatorname{Tx}=\operatorname{Ty} 1(:, 1: m-1) .^{\prime}$;

$Q=T x$;

The Chien search circuit is composed of $t$ multipliers in $G F\left(2^{m}\right)$ and a few adders. It is used to find out the error locations by a full search if $\lambda_{i} \neq 0$.

\section{Related Work}

The popular stream cipher consists of two key stream generators, as is shown in Fig. 2 [18, 19]. The plaintexts are masked by the keys on the left, and then decrypted by the same stream on the right. The symbol 'IV' denotes initialization vector.

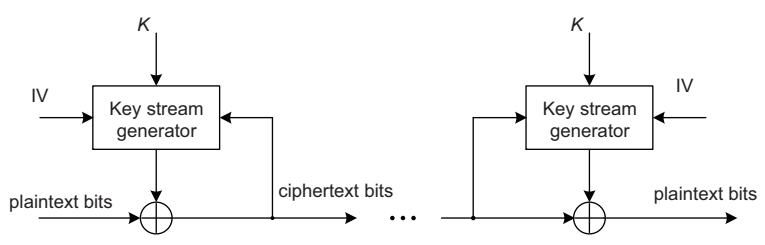

Figure 2: Traditional stream cipher by two key generators.

A random number generator (RNG) can be implemented by the linear feedback shift register (LFSR) [20] that generates peudo-random numbers for stream cipher, as is shown in Fig. 3. In practise the RNG can also be constructed by other digital or analog circuits.

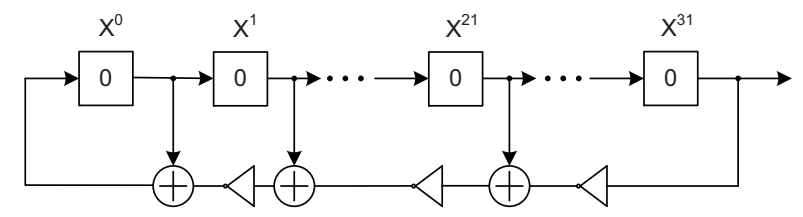

Figure 3: Linear feedback shift registers by XNOR gates[21]. 
A light-weight and energy-friendly stream cipher is proposed for wireless sensor network (WSN) in [18]. The paper suggests use the linear combination of pervious packets to generate the peudo-random key bits in a stream cipher, where a mixed protocol is advanced and public key is used to achieve data privacy [18].

Joint encoding and encryption by LDPC codes and RS codes for public key cryptography is a tradeoff between security and reliable communication [5, 22, 6]. New scrambling and permutating matrices for McEliece system with RS codes are proposed in [6] to enhance security. In [2] the wire-tap encoding and the error correction encoding are concatenated to enhance security. And in [3], a generator matrix, an invertible matrix, and gamma generator are used to produce the ciphertexts.

A provable secure stream cipher based syndrome decoding problem is presented in [7], which uses regular words to speed the system up and quasi-cyclic codes to reduce memory requirements. The results seem to be based on quasi-cyclic BCH codes.

\section{Proposed Approach}

Now suppose the message piece is $r$ and the random numbers $s$ are combined or XORed, then one can encode $(r \oplus \alpha \cdot s)$, where $\alpha$ is the multiple of $s$ or a $u \times v$ matrix with $u=s / w, v=r / w$ and $w$ is the width of a symbol. Next, one piece of the symbols $s$ is inserted by addition modulo $2^{m}$, and the new message reads $r^{\prime}=M+s$. At the other terminal, by RS decoding one can recover $s$ as well as $(r+\alpha s)$. Finally, the $r=(r+\alpha s)-\alpha s$ can be restored. The whole process is shown in Fig. 4 . Especially, the insertion of errors avoids zero bytes by changing them as nonzero bytes within random numbers.

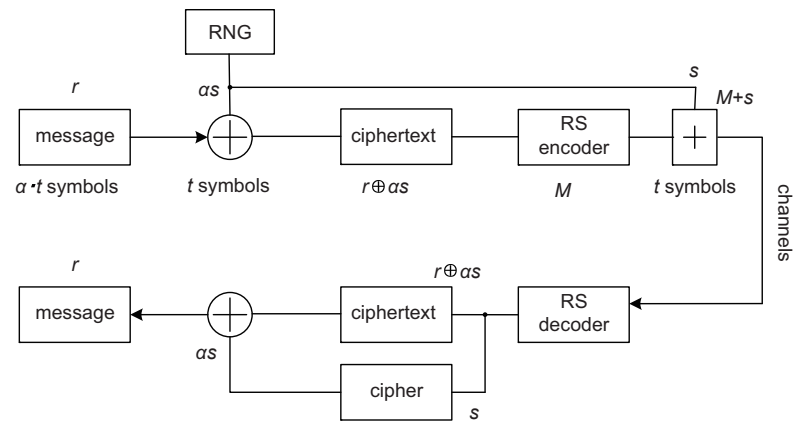

Figure 4: Stream cipher by RS code.

First, the security of a code-based system can be seemed as a public-key security problem, while the usual stream cipher is a symmetric cryptosystem. The proposed mechanism embeds the stream cipher along Reed-Solomon codes looking like a public-key cryptography: considering the key streams as private keys for encoding, and looking at the RS code parameters as the public key to decrypt the ciphertexts.

The security of the proposed scheme may also be improved by the application of shortened codes. In this way, the $n$-symbol codewords are replaced by a $(n-\delta)$-symbol codewords. Take the wireless communication for example, the sender and receiver share the same hardware units, i.e., stream engines, encoders and decoders. Then, the stream cipher with the sender can be seen as private keys to encrypt the messages. The choices of (1) whether a subfield subcode is chosen (2) whether a shortened code is chosen (3) whether the same decoder exists can be used as the public keys. If the decoder exists and the other two parameters are rightly chosen, then the stream cipher can be separated from the message bits by RS decoding.

In detail, one can use subcode or shortened code to decrease the error rate or improve the security of the system, as is shown in Fig. 5. The subfield subcode over $G F\left(p^{s}\right)$ have the same length $n$ but is smaller than its parent code [23] with the word width, which is compressed from $\left\lceil s m \log _{2} p\right\rceil$ to $\left\lceil s \log _{2} p\right\rceil$. In fact, the length $n$ of a subfield subcode may be larger than the size of a subfield, but is constrained its parent field size. Notice that the parity check symbols keep about double word size of other code symbols in Fig. 5.

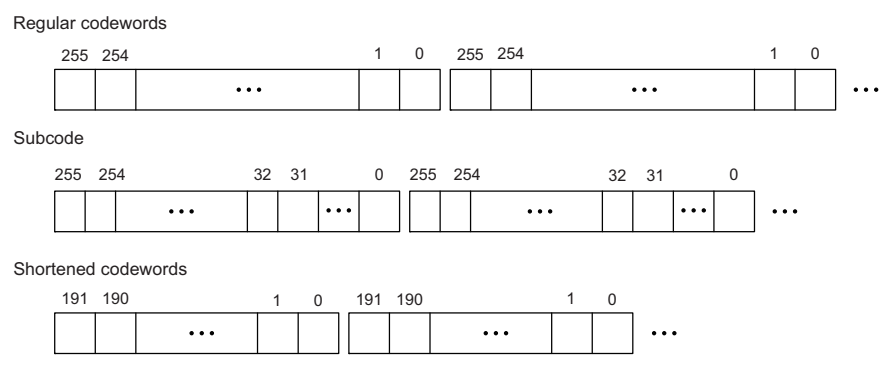

Figure 5: Stream cipher by subcode or shortened RS codes.

Secondly, the proposed scheme reduce the hardware cost and complexity by reusing the encoder for encryption and recovering the key streams by the decoder. Therefore, the task of aligning the stream engines to time sequence becomes unnecessary. As the number of stream cipher engines decreases from 2 to 1 , it reduces the continuous power consumption much. On the one hand, it reduces the hardware units by a half; on the other hand, the renewal frequency of the stream cipher engine can be lowered down. Also, it is not easy to align the messages to real time, but it is feasible to align the messages at code sequence. The oscillator frequency may vary from time to time, while the stream cipher engines requires accurate alignments. The existence of time delay in mobile phones proves the validity of code alignment.

Furthermore, if the function of RS decoding and encoding is occasionally used, then the encryption can be used as an auxiliary function. Otherwise, if the error correction through the transmission path is requirable, then the first few code symbols can be designated as masks of key streams while the next words can be corrected by the remained error correction capability. Since error correction logic and stream cipher are basic units in wireless communications, this scheme 
secret data as stream cipher

$00000000 \mathrm{~h}: 313038$ 3D 33 5E 33 2A 32 5E 32 OD OA OD OA ; $108=3 \wedge 3 * 2 \wedge 2 \ldots$

message

00000000h: $544865205761746572 \quad 204 \mathrm{D} 617267 \quad 696 \mathrm{E}$; THe Water Margin 00000010h: 207465 6C 6C 73 20746865207374 6F 7279 ; tells the story $00000020 \mathrm{~h}: 206 \mathrm{~F} 6620726562656 \mathrm{C} 6 \mathrm{C} 696 \mathrm{~F} 6 \mathrm{E} 20616 \mathrm{E}$; of rebelion an 00000030h: $64207374727567676 \mathrm{C} 65206 \mathrm{~F} 6620736 \mathrm{~B}$; d struggle of sk 00000040h: $696 \mathrm{C} 6 \mathrm{C} 6564$ 2C 206272757465 2C $206 \mathrm{~F} 72$; illed, brute, or 00000050h: $206272617665206 \mathrm{D} 65$ 6E $20696 \mathrm{E} 206163$; brave men in ac $00000060 \mathrm{~h}: 6965$ 6E 74 $204368696 \mathrm{E} 61$ 2E 2054686579 ; ient China. They 00000070h: $20617265206 \mathrm{E} 61$ 6D $6564 \quad 20$ 4A $69616 \mathrm{E} \mathrm{67;} \mathrm{are} \mathrm{named} \mathrm{Jiang}$ 00000080h: 2053 6F 6E 67 2C 20 4A 75 6E 796920 4C 75 2C ; Song, Junyi Lu, 00000090h: $205368656 \mathrm{E} 6720476 \mathrm{~F} 6 \mathrm{E} 677375$ 6E 2E 2E ; Sheng Gongsun. 000000a0h: 2E OD OA OD OA OD OA

message encrypted by stream cipher

00000000h: 6578 5d 1d 64 3f 47 ff 407 e 7 f 6c 78 6a 63 6e; ex]d?GOe lxjen 00000010h: 1144 bd 51 ff 2d 13 5e 5a 3b $127 e 7 e 627879$; $\square$ D] Q_- $\square \wedge$ Z; $\square \sim \sim$ bxy ......

Figure 6: Hiding information as stream cipher in RS codes.

Table 1: Advantages of Proposed Stream Cipher Compared to Other Schemes

\begin{tabular}{|c|c|c|c|c|c|c|c|c|}
\hline Reference & Function & Implement & Topology & $\begin{array}{l}\text { ENC/DEC } \\
\text { Symmetry }\end{array}$ & $\begin{array}{l}\text { Reuse for } \\
\text { ECC }\end{array}$ & No. of RNG & Randomness & Code \\
\hline This work & Stream data & Hardware & 1-to-N & Not full & Yes & 1 & Not strictly & RS \\
\hline 10 & Stream data & Hardware & 1-to-1 & Symmetric & No & 2 & Required & No \\
\hline 7 & Stream data & Software & 1 -to-1 & Symmetric & No & 2 & Required & $\begin{array}{l}\text { Quasi- } \\
\text { cyclic }\end{array}$ \\
\hline 8 & $\begin{array}{l}\text { Exchange } \\
\text { data }\end{array}$ & Software & N-to-N & Not & No & 0 & - & GRS \\
\hline
\end{tabular}

combines their functions.

Thirdly, it may be applicable to transmit extra data through the key streams and therefore increase the throughput of inserted codewords. For example, the $(255,223,16)$ RS code has 223 information symbols. If the encoder are used for stream cipher, then 16 symbols of information can be added to the codeword secretly. The information rate is increased from $223 / 255=0.87$ to $239 / 255=0.94$. As is shown in Fig. 6, it is able to mix information with secret data in the proposed codes.

\section{Comparison}

The comparisons of this work compared with peer works are shown in Tab. 1. It should be noticed that the proposed scheme is not full symmetric, for which the cipher is decoded through the public-key. The architecture is implemented in a topology of 1-to-N, with $N$ being any natural numbers including 1 . If $N=1$, then there are two parties to communicate with each other; or else it can be used in a network. By contrast, the stream cipher is usually supposed to follow 1-to-1 topology. While the encoding and decoding architectures in our work are non-symmetric, the whole stream cipher engine can be made nearly symmetric by integrating an encoder and decoder together. Moreover, this work needs only 1 random number generator while other stream ciphers requires 2 to $N$ random number generators, since the key stream is embedded in the data stream in this work.

The weakly secure data exchange problem with generalized Reed-Solomon code is discussed in [8], in which data exchange between multiple parts rather than two parts are considered.

Also, in the proposal the random number generator is not that strictly required and pseudo random numbers can be used, while other works pay much attention to the random number generator itself, owing to different mechanism for stream ciphers. While traditional stream cipher uses continuous random bits, this work applies data structures of codewords to separate data. The references [8, 7] also uses GRS codes and quasi-cyclic codes to encode data and bring out randomness.

The architecture and hardware implementation results with [10] is demonstrated in Tab. 1 and Tab. 2 . It uses a hardware common key cryptography named RAC for stream cipher, where the random numbers are used as addresses to relocate the data bits. The random numbers are generated by the recipient as addresses to resort the received ciphertext. Assuming the stream cipher engine in [10] works in a pipeline, then it is able to encrypt and decrypt 1 byte every clock cycle with throughput up to $3.2 \mathrm{Gps}$. Nevertheless, considering that the data from the sender or to the recipient should be stored in a RAM for reorganization, so the real decryption throughput may be only a half, let alone the time for fetching instructions.

Finally, the power consciousness of this design is 
demonstrated by the application of RS code, whereas McEliece systems usually use nonsystematic encoding with an increase of computational efforts by $n /(n-k)$ times. In addition, if there are demands of error corrections for channels, the hardware units may be reconfigured and reused at different times.

Table 2: Hardware Implementation of Stream Cipher Engine by Reed-Solomon Code.

\begin{tabular}{lllll}
\hline Design & Platform & LUTs & $\begin{array}{l}f_{\max } \\
(\mathrm{MHz})\end{array}$ & $\begin{array}{l}\text { Throughput } \\
(\mathrm{Gps})\end{array}$ \\
\hline Enc & XC5VLX85-2 & 318 & 480 & 3.79 \\
Dec & XC5VLX85-2 & 5253 & 270 & 0.289 \\
Enc/Dec & XC5VLX85-2 & 5905 & 270 & $1.82 / 0.289$ \\
10 & $0.18 \mu \mathrm{m} \mathrm{CMOS}$ & $1.38 \mathrm{~mm}^{2}$ & 400 & $1.6 \sim 3.2$ \\
\hline
\end{tabular}

The stream cipher with encoding and decoding by RS codes is described by Matlab for test at first, then it is described by Verilog HDL for hardware implementation. As is shown in Tab. 2, the stream cipher engine is simulated by Modelsim 6.2, synthesized by Synopsys Synplify Pro 2014, and placed and rooted in Xilinx ISE 14.7. The code parameters are chosen as usual in [24], i.e.,

$m=8$ : number of bits per symbol,

$n=255$ : number of symbols per codeword,

$k=223$ : number of message symbols in a codeword,

$t=16$ : number of corrected errors by symbols.

According to the theory and results in [7], the security level of the cipher in the above table is about 80 bits of keys with symmetric cryptography.

In Tab. 2, the RS code supports correcting 16 symbols of errors along with 223 symbols of messages. It takes about $3.78 \mu$ s to correct 2 errors of symbols, and $7.06 \mu$ s to correct 16 errors of symbols. Its information throughput without parity check bits are 1.59/0.253 Gbit/s respectively for encoding and decoding.

\section{Conclusion}

Stream cipher is often used for mobile communications between two parties. In general, this paper proposes an easy scheme to implement stream cipher along with the popular RS code, which relies on RS encoding and decoding to cover and uncover the key streams. It is beneficial to easily evaluate the complexity and enhance the security of communication systems. The proposed cipher is based on the NP-complete problem of decoding a random linear code, which simply conforms to many solved code-based security issues. The cipher also decreases one key generator and reduces unnecessary power consumption for the synchronization between parties.

\section{Acknowledgment}

The author would like to appreciate the editor and reviewers for their kind help. This work is supported by Shenzhen postdoctoral base for innovation and prac- tice and partly by Shenzhen basic research program (No. JCYJ20170307141601162).

\section{References}

[1] T. Wu and R. Wang, "Stream cipher by Reed-Solomon code," in International Conference on Information and Communication Technology Convergence (ICTC), 2017, pp. 422-427.

[2] F. E. Oggier and M. J. Mihaljevic, "An information-theoretic analysis of the security of communication systems employing the encoding-encryption paradigm," arXiv, 2018. [Online]. Available: http://arxiv.org/abs/1008.0968

[3] A. N. Alekseychuk and S. V. Gryshakov, "Secure and practical randomized stream ciphers based on ReedSolomon codes," Cybernetics and Systems Analysis, vol. 53, no. 2, pp. 262-268, 2017.

[4] S. Lin and J. Daniel J. Costello, Error Control Coding. Upper Saddle River, New Jersey: Pearson Education, Inc., 2004.

[5] M. Baldi, QC-LDPC Code-Based Cryptography, ser. Briefs in Electrical and Computer Engineering. Springer, 2014.

[6] M. Baldi, F. Chiaraluce, M. Bianchi, and J. R. D. Schipani, "Enhanced public key security for the McEliece cryptosystem," Journal of Cryptology, vol. 29, no. 1, p. 127, January 2016.

[7] P. Gaborit, C. Lauradoux, and N. Sendrier, "SYND: a fast codebased stream cipher with a security reduction," in IEEE International Symposium on Information Theory, 2007, pp. 186-190.

[8] M. Yan, A. Sprintson, and I. Zelenkoy, "Weakly secure data exchange with generalized reed solomon codes," in IEEE International Symposium on Information Theory, 2014, pp. 1366-1370.

[9] M. Orumiehchiha, J. Pieprzyk, E. Shakour, and R. Steinfeld, "Security evaluation of Rakaposhi stream cipher," in International Conference on Information Security Practice and Experience (ISPEC 2013), ser. Lecture Notes in Computer Science, R. Deng and T. Feng, Eds., vol. 7863. Berlin, Heidelberg: Springer, 2013, pp. 361-371.

[10] M. Fukase, H. Takeda, R. Tenma, K. Noda, Y. Sato, R. Sato, and T. Satot, "Development of a multimedia stream cipher engine," in International Symposium on Intelligent Signal Processing and Communication Systems (ISPACS2006), Tottori, Japan, 2006, pp. 562-565.

[11] H. C. van Tilborg, Fundamentals of cryptology: A Professional Reference and Interactive Tutorial. Boston/Dordrecht/London: Kluwer Academic Publishers, 1999.

[12] T. Eisenbarth, T. Guneysu, S. Heyse, and C. Paar, "Microeliece: Mceliece for embedded devices," in CHES, ser. LNCS, vol. 5747, 2009 , p. 4964.

[13] S. Heyse, I. von Maurich, and T. Güneysu, "Smaller keys for code-based cryptography: QC-MDPC McEliece implementations on embedded devices," in CHES, ser. LNCS, vol. 8086, 2013, pp. 273-292.

[14] S. Lin and J. Daniel J. Costello, Error Control Coding- Fundamentals and Applications. New Jersey: Prentice Hall, 1983.

[15] B. Sklar, "Reed-solomon codes," 2001. [Online]. Available: http://ptgmedia.pearsoncmg.com/images/art_sklar7_ reed-solomon/elementLinks/art_sklar7_reed-solomon.pdf

[16] D. V. Sarwate and N. R. Shanbhag, "High-speed architectures for reedsolomon decoders," IEEE Transactions on VLSI Systems, vol. 9, no. 5, pp. 641-655, October 2001.

[17] A. Reyhani-Masoleh and M. A. Hasan, "Low complexity bit parallel architectures for polynomial basis multiplication over GF $\left(2^{m}\right)$," IEEE Transactions on Computers, pp. 945-959, 2004.

[18] H. Lin, A. J. A., D. Bai, and Y. Liu, "A light-weight stream cipher for wireless sensor networks," in Future Wireless Networks and Information Systems, ser. Lecture Notes in Electrical Engineering, Y. Zhang, Ed., vol. 143. Berlin, Heidelberg: Springer, 2012, pp. 627-638.

[19] B. Sklar, Digital Communications Fundamentals and Applications. Beijing: Publishing House of Electronics Industry, 2015, in Chinese.

[20] A. Canteaut, "Linear feedback shift register," pp. 726-729, 2011, reference Work Entry. 
[21] "Linear feedback shift registers in virtex devices," 1996. [Online]. Available: http://www.xilinx.com/support/ documentation/application_notes/xapp052.pdf

[22] M. Esmaeili and T. A. Gulliver, "Joint channel codingcryptography based on random insertions and deletions in quasi-cyclic-low-density parity check codes," IET Communications, vol. 9, no. 12, pp. 1555-1560, 2015.
[23] J. I. Hall, http://users.math.msu.edu/users/jhall/classes/ codenotes/Subfields.pdf Michigan State University, pp. 8999, 2012, codes over Subfields.

[24] Z. Liang and W. Zhang, "Efficient Berlekamp-Massey algorithm and architecture for Reed-Solomon decoder," Journal of Signal Processing Systems, vol. 86, p. 5165, 2017. 